\title{
Determination of an Unknown Source in the Heat Equation by the Method of Tikhonov Regularization in Hilbert Scales
}

\author{
Zhenyu Zhao ${ }^{1}$, Ou Xie ${ }^{1}$, Zehong Meng ${ }^{2}$, Lei You ${ }^{1}$ \\ ${ }^{1}$ College of Science, Guangdong Ocean University, Zhanjiang, China \\ ${ }^{2}$ School of Mathematics and Statistics, Zhejiang University of Finance and Economics, Hangzhou, China \\ Email: dongdongzb@gmail.com
}

Received December 9, 2013; revised January 9, 2014; accepted January 15, 2014

Copyright (C) 2014 Zhenyu Zhao et al. This is an open access article distributed under the Creative Commons Attribution License, which permits unrestricted use, distribution, and reproduction in any medium, provided the original work is properly cited. In accordance of the Creative Commons Attribution License all Copyrights (C) 2014 are reserved for SCIRP and the owner of the intellectual property Zhenyu Zhao et al. All Copyright (C) 2014 are guarded by law and by SCIRP as a guardian.

\section{ABSTRACT}

In this paper, we consider the problem for determining an unknown source in the heat equation. The Tikhonov regularization method in Hilbert scales is presented to deal with ill-posedness of the problem and error estimates are obtained with a posteriori choice rule to find the regularization parameter. The smoothness parameter and the a priori bound of exact solution are not needed for the choice rule. Numerical tests show that the proposed method is effective and stable.

\section{KEYWORDS}

Ill-Posed Problem; Unknown Source; Heat Equation; Regularization Method; Discrepancy Principle in Hilbert Scales

\section{Introduction}

In this paper, we consider the following problem for determining the unknown source in the heat equation [1]:

$$
\left\{\begin{array}{l}
u_{t}=u_{x x}+f(x), x \in \mathbb{R}, 0<t<1, \\
u(x, 0)=0, \quad x \in \mathbb{R} \\
u(x, 1)=g(x), \quad x \in \mathbb{R}
\end{array}\right.
$$

where $u(\cdot, t) \in L^{2}(\mathbb{R})$ represents state variable. Our purpose is to identify the source term $f(x)$ from the data $u(x, 1)=g(x)$. This problem is called the inverse source problem. In practice, the data at $g(x)$ are often obtained on the basis of reading of physical instrument. So only the perturbed data $g^{\delta}(x)$ can be obtained. We assume that the exact and measured data satisfy

$$
\left\|g-g^{\delta}\right\| \leq \delta
$$

where $\delta>0$ denotes the noisy level, $\|\cdot\|$ denotes the $L^{2}$-norm.

A variety of important problems in science and engineering involve the inverse source problems, e.g. heat conduction, crack identification electromagnetic theory, geophysical prospecting and pollutant detection. These problems are well known to be ill posed (the solution, if it exists, does not depend continuously on the data). Thus, the numerical simulation is very difficult and some special regularization is required. A few papers have presented the mathematical analysis and effective algorithms of these problems. The uniqueness and conditional stability results for these problems can be found in [2-7]. Some numerical reconstruction schemes can be found in [1,8-17]. 
In [1], the optimal error bound of the problem (1.1) has been obtained and a Fourier regularization method with an a prior parameter choice rule has been presented. It is well known that the ill posed problem is usually sensitive to the regularization parameter and the a priori bound is difficult to be obtained precisely in practice. In [18], we have used a modified Tikhonov regularization method with an a posteriori choice to solve the problem. But the smoothness parameter (which is usually unknown) is needed for that method. In this paper, we will use the method of Tikhonov regularization in Hilbert scales to solve the problem. We will show that the regularization parameter can be chosen by a discrepancy principle in Hilbert scales which is proposed by Neubauer [19]. The smoothness parameter and the a priori bound of exact solution are not needed for the new method.

This paper is organized as follows. In Section 2, we will give the method of Tikhonov regularization in Hilbert scales for the problem (1.1). The choice of regularization parameter and corresponding convergence results will be found in Section 3. Some numerical results are given in Section 4 to show the effectiveness of the new method.

\section{The Method of Tikhonov Regularization in Hilbert Scalles for the Problem (1.1)}

Let $\hat{h}$ denote the Fourier transform of $h \in L^{2}(\mathbb{R})$ defined by

$$
\hat{h}(\xi)=\mathscr{F}[h(x)]=\frac{1}{\sqrt{2 \pi}} \int_{-\infty}^{+\infty} h(x) \mathrm{e}^{-\mathrm{i} \xi x} \mathrm{~d} x,
$$

and $\|\cdot\|_{p}$ denotes the norm in Sobolev space $H^{p}(\mathbb{R})$ defined by

$$
\|h\|_{p}:=\left(\int_{-\infty}^{+\infty}\left(1+\xi^{2}\right)^{p}|\hat{h}(\xi)|^{2} \mathrm{~d} \xi\right)^{1 / 2} .
$$

When $p=0,\|\cdot\|_{0}=:\|\cdot\|$ denotes the $L^{2}(\mathbb{R})$ norm.

Application of the Fourier transform technique to problem (1.1) with respect to the variable $x$ yields the following problem in the frequency space:

$$
\begin{cases}\hat{u}_{t}(\xi, t)+\xi^{2} \hat{u}(\xi, t)=\hat{f}(\xi), & \xi \in \mathbb{R}, 0<t<1, \\ \hat{u}(\xi, 0)=0, & \xi \in \mathbb{R}, \\ \hat{u}(\xi, 1)=\hat{g}(\xi), & \xi \in \mathbb{R} .\end{cases}
$$

It is easy to see that the solution of problem (1.5) is

$$
\hat{f}(\xi)=\lambda(\xi) \hat{g}(\xi)
$$

where

$$
\lambda(\xi)=\frac{\xi^{2} \mathrm{e}^{\xi^{2}}}{\mathrm{e}^{\xi^{2}}-1},
$$

or equivalently, the solution of problem (1.1) is given by

$$
f(x)=\mathscr{F}^{-1}[\lambda(\xi) \hat{g}(\xi)]=\frac{1}{\sqrt{2 \pi}} \int_{-\infty}^{\infty} \frac{\xi^{2} \mathrm{e}^{\xi^{2}}}{\mathrm{e}^{\xi^{2}}-1} \hat{g}(\xi) \mathrm{e}^{\mathrm{i} \xi x} \mathrm{~d} x=: T g .
$$

It is apparent that the exact data $\hat{g}$ must decay faster than the rate $|\xi|^{-2}$. However, in general, the measured data $g^{\delta}$ does not possess such a decay property. In the following, we apply the Tikhonov regularization method to reconstruct a new function $\varphi^{\delta}$ from the perturbed data $g^{\delta}$. T $\varphi^{\delta}$ will give a reliable approximation of $f$. Before doing that, we impose an a priori bound on the unknown source

$$
\|f\|_{p} \leq E, p \geq 0 .
$$

In this case, we let $\varphi^{\delta}=\varphi^{\alpha, \delta}$ be the minimizer of the Tikhonov functional

$$
\Phi(\varphi)=\left\|\varphi-g^{\delta}\right\|^{2}+\alpha\|T \varphi\|_{q}^{2},
$$

where $\alpha>0$ is a regularization parameter and $q$ is a positive real number. It can be verified that $\varphi^{\alpha, \delta}$ is the 
solution of the following equation [20]

$$
\left(I+\alpha \mathscr{F}^{-1}\left[\left(1+\xi^{2}\right)^{q} \lambda(\xi)^{2}\right] \mathscr{F}\right) \varphi=g^{\delta} .
$$

So we can get

$$
\hat{\varphi}^{\alpha, \delta}=\frac{1}{1+\alpha\left(1+\xi^{2}\right)^{q} \lambda(\xi)^{2}} \hat{g}^{\delta} .
$$

That is to say

$$
\varphi^{\alpha, \delta}=\mathscr{F}^{-1}\left[\frac{1}{1+\alpha\left(1+\xi^{2}\right)^{q} \lambda(\xi)^{2}} \hat{g}^{\delta}\right] .
$$

By $\varphi^{\alpha, \delta}$, we can give an approximation of $f$ as follows:

$$
f^{\alpha, \delta}=T \varphi^{\alpha, \delta}=\mathscr{F}^{-1}\left[\frac{\lambda(\xi)}{1+\alpha\left(1+\xi^{2}\right)^{q} \lambda(\xi)^{2}} \hat{g}^{\delta}\right] .
$$

Lemma 1 For any $\xi \in \mathbb{R}$, we have

$$
0<\lambda(\xi) \leq 1+\xi^{2} \leq 2 \lambda(\xi), \xi \in \mathbb{R} .
$$

Lemma 2 [21] For $0 \leq b \leq 1$, we have

$$
\sup _{\lambda \geq 0} \frac{\lambda^{b}}{\lambda+\alpha}=b^{b}(1-b)^{1-b} \alpha^{b-1} .
$$

\section{Lemma 3}

$$
\left\|f^{\alpha, \delta}-T \varphi^{\alpha}\right\|=O\left(\delta \cdot \alpha^{-1 /(q+2)}\right)
$$

where $\varphi^{\alpha}$ is the unique minimizer of (1.10) with $g$ instead of $g^{\delta}$.

Proof 1 Due to Parseval formula and Lemma 1

$$
\begin{aligned}
\left\|f^{\alpha, \delta}-T \varphi^{\alpha}\right\|^{2} & =\int_{-\infty}^{\infty}\left|\frac{\lambda(\xi)}{1+\alpha\left(1+\xi^{2}\right)^{q} \lambda(\xi)^{2}}\left(\hat{g}^{\delta}-\hat{g}\right)\right|^{2} \mathrm{~d} \xi \leq \int_{-\infty}^{\infty}\left|\frac{\lambda(\xi)}{1+\alpha \lambda(\xi)^{q+2}}\left(\hat{g}^{\delta}-\hat{g}\right)\right|^{2} \mathrm{~d} \xi \\
& =\int_{-\infty}^{\infty}\left|\frac{\lambda(\xi)^{-q-1}}{\lambda(\xi)^{-q-2}+\alpha}\left(\hat{g}^{\delta}-\hat{g}\right)\right|^{2} \mathrm{~d} \xi \leq\left(\sup _{\lambda \geq 0} \frac{\lambda^{\frac{q+1}{q+2}}}{\lambda+\alpha}\right) \int_{-\infty}^{\infty}\left|\left(\hat{g}^{\delta}-\hat{g}\right)\right|^{2} \mathrm{~d} \xi
\end{aligned}
$$

The proposition follows by applying (1.16) with $b$ replaced by $\frac{q+1}{q+2}$.

\section{Lemma 4}

$$
\left\|T \varphi^{\alpha}-f\right\| \leq c_{\alpha} \cdot \alpha^{\frac{p}{2(q+2)}} .
$$

where $c_{\alpha}$ is defined by

$$
c_{\alpha}^{2}:=\int_{-\infty}^{\infty} \frac{\alpha^{2-\frac{p}{q+2}}}{\left(\left(1+\xi^{2}\right)^{-q-2}+\alpha\right)^{2}}|\hat{f}(\xi)|^{2} \mathrm{~d} \xi .
$$

Proof 2 With the representation

$$
T \varphi^{\alpha}=\mathscr{T}^{-1}\left[\frac{1}{1+\alpha\left(1+\xi^{2}\right)^{q} \lambda(\xi)^{2}} \hat{f}(\xi)\right] .
$$


and Lemma 1, we have

$$
\begin{aligned}
\left\|T \varphi^{\alpha}-f\right\|^{2} & =\int_{-\infty}^{\infty}\left|\frac{\alpha\left(1+\xi^{2}\right)^{q} \lambda(\xi)^{2}}{1+\alpha\left(1+\xi^{2}\right)^{q} \lambda(\xi)^{2}} \hat{f}(\xi)\right|^{2} \mathrm{~d} \xi \leq \int_{-\infty}^{\infty}\left(\frac{\alpha\left(1+\xi^{2}\right)^{q+2}}{1+\alpha\left(1+\xi^{2}\right)^{q+2}}\right)^{2}|\hat{f}(\xi)|^{2} \mathrm{~d} \xi \\
& =\int_{-\infty}^{\infty}\left(\frac{\alpha}{\left(1+\xi^{2}\right)^{-(q+2)}+\alpha}\right)|\hat{f}(\xi)|^{2} \mathrm{~d} \xi \\
& =\alpha^{\frac{p}{q+2}} \int_{-\infty}^{\infty} \frac{\alpha^{2-\frac{p}{q+2}}}{\left(\left(1+\xi^{2}\right)^{-q-2}+\alpha\right)^{2}}|\hat{f}(\xi)|^{2} \mathrm{~d} \xi .
\end{aligned}
$$

\section{The Choice of Regularization Parameter $\alpha$ and Convergence Results}

For any $w \in L^{2}(\mathbb{R})$, we define

$$
d(\alpha, w):=\int_{-\infty}^{\infty}\left(\frac{\alpha}{\left(1+\xi^{2}\right)^{-(q+2)}+\alpha}\right)^{2}|\hat{w}(\xi)|^{2} \mathrm{~d} \xi
$$

It is apparent that the function $\alpha \rightarrow d(\alpha, w)$ is continuous and strictly increasing on $(0, \infty)$ and

$$
\lim _{\alpha \rightarrow 0} d(\alpha, w)=0, \quad \lim _{\alpha \rightarrow \infty} d(\alpha, w)=\|w\|^{2}
$$

So we can get the following lemma.

Lemma 5 Let $g, g^{\delta}$ and $\delta>0$ satisfy (1.2) and

$$
\left\|g^{\delta}\right\| \geq C \delta
$$

for some $C>1$. Then there is a unique $\alpha>0$ such that

$$
d\left(\alpha, g^{\delta}\right)=C \cdot \delta^{2} .
$$

In the following, we denote the unique $\alpha$ determined in (1.26) by $\bar{\alpha}$. In the next lemma we consider the behavior of $\bar{\alpha}$.

Lemma 6 Let $g, g^{\delta}$ and $\delta>0$ satisfy (1.2) and (1.25) for a $C>1$, then

1)

$$
d(\bar{\alpha}, g)=O\left(\delta^{2}\right)
$$

2)

$$
\bar{\alpha} \leq 4 \delta^{\frac{2(q+2)}{p+2}} \cdot d_{\bar{\alpha}}^{-\frac{2(q+2)}{p+2}}
$$

where

$$
d_{\bar{\alpha}}^{2}=\int_{-\infty}^{\infty} \frac{\bar{\alpha}^{2-\frac{p+2}{q+2}}\left(1+\xi^{2}\right)^{-2}}{\left[\left(1+\xi^{2}\right)^{-(q+2)}+\bar{\alpha}\right]^{2}}|\hat{f}(\xi)|^{2} \mathrm{~d} \xi
$$

Proof 1) Let

$$
L w=\mathscr{F}^{-1}\left(\frac{\bar{\alpha}}{\left(1+\xi^{2}\right)^{-(q+2)}+\bar{\alpha}} \hat{w}(\xi)\right)
$$


then

$$
d(\bar{\alpha}, g)=\|L g\|^{2} \leq\left(\left\|L\left(g-g^{\delta}\right)\right\|+\left\|L g^{\delta}\right\|\right)^{2} \leq(\delta+\sqrt{C} \delta)^{2}=(1+\sqrt{C})^{2} \delta^{2}
$$

2)

$$
\begin{aligned}
d(\bar{\alpha}, g) & =\int_{-\infty}^{\infty}\left(\frac{\bar{\alpha}}{\left(1+\xi^{2}\right)^{-(q+2)}+\bar{\alpha}}\right)^{2}|\hat{g}(\xi)|^{2} \mathrm{~d} \xi \\
& =\int_{-\infty}^{\infty} \frac{\bar{\alpha}^{2}}{\left[\left(1+\xi^{2}\right)^{-(q+2)}+\bar{\alpha}\right]^{2} \lambda(\xi)^{2}}|\hat{f}(\xi)|^{2} \mathrm{~d} \xi \leq\left. 4 \int_{-\infty}^{\infty} \frac{\bar{\alpha}^{2}\left(1+\xi^{2}\right)^{-2}}{\left[\left(1+\xi^{2}\right)^{-(q+2)}+\bar{\alpha}\right]^{2}} \hat{f}(\xi)\right|^{2} \mathrm{~d} \xi \\
& =4 \bar{\alpha}^{\frac{p+2}{q+2}} \int_{-\infty}^{\infty} \frac{\bar{\alpha}^{2-\frac{p+2}{q+2}}\left(1+\xi^{2}\right)^{-2}}{\left[\left(1+\xi^{2}\right)^{-(q+2)}+\bar{\alpha}\right]^{2}}|\hat{f}(\xi)|^{2} \mathrm{~d} \xi
\end{aligned}
$$

The rest follows from 1).

Now we can prove the main result of this paper.

Theorem 1 Let $g, g^{\delta}$ and $\delta>0$ satisfy (1.2) and (1.25) for a $C>1, \quad s \in \mathbb{R} . f^{\bar{\alpha}, \delta}$ is defined by (1.14) with the regularization parameter $\bar{\alpha}$ chosen in (1.26), then

$$
\left\|f^{\bar{\alpha}, \delta}-f\right\|=\left\{\begin{array}{l}
o\left(\delta^{\frac{p}{p+2}}\right) \text { if } p<2 q+2, \\
O\left(\delta^{\frac{p}{p+2}}\right) \text { if } p=2 q+2 .
\end{array}\right.
$$

Proof 4 With Lemma 3, Lemma 4, Lemma 6, we obtain

$$
\begin{aligned}
\left\|f^{\bar{\alpha}, \delta}-f\right\| & \leq\left\|f^{\bar{\alpha}, \delta}-f^{\bar{\alpha}}\right\|+\left\|f^{\bar{\alpha}}-f\right\|=O\left(\delta \cdot \bar{\alpha}^{-1 /(q+2)}\right)+O\left(c_{\alpha} \cdot \bar{\alpha}^{\frac{p}{2(q+2)}}\right) \\
& =O\left[\delta^{\frac{p}{p+2}}\left(d_{\bar{\alpha}}^{\frac{2}{p+2}}+c_{\bar{\alpha}} d_{\bar{\alpha}}^{\frac{-p}{p+2}}\right)\right]
\end{aligned}
$$

Using the Hölder inequality, (1.20) and (1.29) we get

$$
\begin{aligned}
c_{\alpha}^{2} & =\int_{-\infty}^{\infty} \frac{\alpha^{2-\frac{p}{q+2}}}{\left(\left(1+\xi^{2}\right)^{-q-2}+\alpha\right)^{2}}|\hat{f}(\xi)|^{2} \mathrm{~d} \xi \\
& =\int_{-\infty}^{\infty}\left[\frac{\alpha^{2-\frac{p+2}{q+2}}\left(1+\xi^{2}\right)^{-2}}{\left[\left(1+\xi^{2}\right)^{-q-2}+\alpha\right]^{2}}|\hat{f}(\xi)|^{2}\right]^{\frac{p}{p+2}}\left[\left(\frac{\alpha}{\left(1+\xi^{2}\right)^{-q-2}+\alpha}\right)^{2}\left(1+\xi^{2}\right)^{p}|\hat{f}(\xi)|^{2}\right]^{\frac{2}{p+2}} \mathrm{~d} \xi \\
& \leq\left(d_{\alpha}^{2}\right)^{\frac{p}{p+2}} \cdot\left[\int_{-\infty}^{\infty}\left(\frac{\alpha}{\left(1+\xi^{2}\right)^{-q-2}+\alpha}\right)^{2}\left(1+\xi^{2}\right)^{p}|\hat{f}(\xi)|^{2} \mathrm{~d} \xi\right]^{\frac{2}{p+2}}
\end{aligned}
$$

Combining (1.34) and (1.35), we obtain 


$$
\left\|f^{\bar{\alpha}, \delta}-f\right\|=O\left[\delta^{\frac{p}{p+2}}\left(d_{\bar{\alpha}}^{\frac{2}{p+2}}+e_{\bar{\alpha}}^{\frac{2}{p+2}}\right)\right]
$$

where $e_{\alpha}$ is defined by

$$
e_{\alpha}=\int_{-\infty}^{\infty}\left(\frac{\alpha}{\left(1+\xi^{2}\right)^{-q-2}+\alpha}\right)^{2}\left(1+\xi^{2}\right)^{p}|\hat{f}(\xi)|^{2} \mathrm{~d} \xi
$$

Since $\bar{\alpha} \rightarrow 0$ for $\delta \rightarrow 0$ and

$$
\begin{aligned}
& e_{\alpha}=o(1), \text { for } \alpha \rightarrow 0 \\
& d_{\alpha}=\left\{\begin{array}{l}
o(1) \text { if } p<2 q+2 \\
O(1) \text { if } p=2 q+2
\end{array} \text { for } \alpha \rightarrow 0\right.
\end{aligned}
$$

The theorem is proved.

\section{Numerical Examples}

The proposed method can be easily implemented numerically by the fast Fourier transform. We consider the following example.

Example[1] It is easy to verify that the pair of functions

$$
\begin{gathered}
u(x, t)=\frac{x}{(t+1)^{\frac{3}{2}}} \exp \left(-\frac{x^{2}}{4(t+1)}\right)-x \exp \left(-\frac{x^{2}}{4}\right), \\
f(x)=\left(\frac{x^{3}}{4}-\frac{3 x}{2}\right) \exp \left(-\frac{x^{2}}{4}\right)
\end{gathered}
$$

is the exact solution of problem (1.1) with data

$$
g(x)=\frac{x}{2^{\frac{3}{2}}} \exp \left(-\frac{x^{2}}{8}\right)-x \exp \left(-\frac{x^{2}}{4}\right)
$$

Since $f(x)$ approaches zero as $|x|>6$, we always fix the interval $-10 \leq x \leq 10$ in the numerical experiment. Let $x_{j}=-10+j h, j=0,1, \cdots, N-1, N=256$. The perturbed data are given by

$$
g^{\delta}\left(x_{j}\right)=g\left(x_{j}\right)+\varepsilon_{j},
$$

where $\left\{\varepsilon_{j}\right\}_{j=0}^{N}$ are generated by Function $\operatorname{rand} n(N, 1) \times \delta_{1}$ in Matlab.

In the following, we present numerical results to check the efficiency of the method. In the following, we present numerical results of some examples to check the efficiency of the method and we will also compare the

method (M1) with the method in [18] (M2, notate the approximate function as $\left.f^{\alpha, \delta}\right)$.
It is obvious that the condition (1.9) holds for any $p \in \mathbb{R}^{+}$. So we have $\left\|f^{\alpha, \delta}-f\right\|=O\left(\delta^{\frac{q+1}{q+2}}\right), \forall q>0$ for M1 and $\left\|f^{\alpha, \delta}-f\right\|=O\left(\delta^{\frac{q}{q+2}}\right)$ for M2. The relative error has been displayed in Table 1 , we can see that when $\delta_{1}$ decreases from 0.1 to 0.0001 , the errors $\left\|f^{\bar{\alpha}, \delta}-f\right\|$ become smaller and the results of M1 are better than M2.

\section{Conclusion}

In this paper, we present a modified Tikhonov regularization method for identifying an unknown source in the heat equation and the theoretical results show that the method is Order optimal. The numerical example also verified the efficiency and accuracy of the method. 
Table 1. Numerical results with a posteriori parameter.

\begin{tabular}{|c|c|c|c|c|c|c|c|c|}
\hline \multirow{2}{*}{$\delta_{1}$} & \multicolumn{2}{|c|}{$q=0$} & \multicolumn{2}{|c|}{$q=2$} & \multicolumn{2}{|c|}{$q=4$} & \multicolumn{2}{|c|}{$q=8$} \\
\hline & M1 & M2 & M1 & M2 & M1 & M2 & M1 & M2 \\
\hline $1 e^{-1}$ & $1.73 e^{-1}$ & $2.33 e^{-1}$ & $9.18 e^{-2}$ & $1.91 \mathrm{e}^{-1}$ & $8.68 \mathrm{e}-2$ & $1.89 \mathrm{e}-1$ & $8.90 \mathrm{e}-2$ & $1.85 \mathrm{e}^{-1}$ \\
\hline $1 e^{-2}$ & $6.61 \mathrm{e}^{-2}$ & $6.73 e^{-2}$ & $1.81 \mathrm{e}^{-2}$ & $2.60 e^{-2}$ & $1.54 \mathrm{e}^{-2}$ & $2.34 \mathrm{e}^{-2}$ & $1.47 e^{-2}$ & $2.55 e^{-2}$ \\
\hline $1 e^{-3}$ & $1.98 e^{-2}$ & $2.35 e^{-}-2$ & $2.91 e^{-3}$ & $4.85 e^{-3}$ & $2.24 \mathrm{e}^{-}-3$ & $3.48 e^{-}-3$ & $1.98 e^{-3}$ & $3.54 e^{-3}$ \\
\hline $1 e^{-4}$ & $7.8029 e^{-3}$ & $8.79 e^{-3}$ & $5.99 e^{-4}$ & $8.57 e^{-4}$ & $2.81 e^{-4}$ & $5.78 e^{-4}$ & $2.25 e^{-4}$ & $5.53 e^{-4}$ \\
\hline
\end{tabular}

\section{Acknowledgements}

The project is supported by the National Natural Science Foundation of China (No.11201085).

\section{REFERENCES}

[1] F. F. Dou, C. L. Fu and F. L. Yang, "Optimal Error Bound and Fourier Regularization for Identifying an Unknown Source in the Heat Equation,” Journal of Computational and Applied Mathematics, Vol. 230, No. 2, 2009, pp. 728-737. http://dx.doi.org/10.1016/j.cam.2009.01.008

[2] J. R. Cannon and P. Du Chateau, "Structural Identification of an Unknown Source term in a Heat Equation,” Inverse Problems, Vol. 14, No. 3, 1998, pp. 535-551. http://dx.doi.org/10.1088/0266-5611/14/3/010

[3] J. R. Cannon and S. Perez-Esteva, “Uniqueness and Stability of 3d Heat Sources,” Inverse Problems, Vol. 7, No. 1, 1991, pp. 57-62. http://dx.doi.org/10.1088/0266-5611/7/1/006

[4] M. Choulli and M. Yamamoto, “Conditional Stability in Determining a Heat Source,” Journal of Inverse and Ill-Posed Problems, Vol. 12, No. 3, 2004, pp. 233-243. http://dx.doi.org/10.1515/1569394042215856

[5] A. El Badia and T. Ha-Duong, "On an Inverse Source Problem for the Heat Equation. Application to a Pollution Detection Problem,” Journal of Inverse and Ill-Posed Problems, Vol. 10, No. 6, 2002, pp. 585-600. http://dx.doi.org/10.1515/jiip.2002.10.6.585

[6] G. S. Li, "Data Compatibility and Conditional Stability for an Inverse Source Problem in the Heat Equation,” Applied Mathematics and Computation, Vol. 173, No. 1, 2006, pp. 566-581. http://dx.doi.org/10.1016/j.amc.2005.04.053

[7] M. Yamamoto, “Conditional Stability in Determination of Force Terms of Heat Equations in a Rectangle," Mathematical and Computer Modelling, Vol. 18, No. 1, 1993, pp. 79-88. http://dx.doi.org/10.1016/0895-7177(93)90081-9

[8] A. A. Burykin and A. M. Denisov, "Determination of the Unknown Sources in the Heat-Conduction Equation," Computational Mathematics and Modeling, Vol. 8, No. 4, 1997, pp. 309-313. http://dx.doi.org/10.1016/0895-7177(93)90081-9

[9] A. Farcas and D. Lesnic, "The Boundary-Element Method for the Determination of a Heat Source Dependent on One Variable," Journal of Engineering Mathematics, Vol. 54, No. 4, 2006, pp. 375-388. http://dx.doi.org/10.1007/s10665-005-9023-0

[10] L. Ling, M. Yamamoto, Y. C. Hon and T. Takeuchi, “Identification of Source Locations in Two-Dimensional Heat Equations,” Inverse Problems, Vol. 22, No. 4, 2006, pp. 1289-1305. http://dx.doi.org/10.1088/0266-5611/22/4/011

[11] H. M. Park and J. S. Chung, “A Sequential Method of Solving Inverse Natural Convection Problems,” Inverse Problems, Vol. 18, No. 3, 2002, pp. 529-546. http://dx.doi.org/10.1088/0266-5611/18/3/302

[12] V. S. Ryaben'kii, S. V. Tsynkov and S. V. Utyuzhnikov, "Inverse Source Problem and Active Shielding for Composite Domains,” Applied Mathematics Letters, Vol. 20, No. 5, 2007, pp. 511-515. http://dx.doi.org/10.1016/j.aml.2006.05.019

[13] L. Yan, C. L. Fu and F. L. Yang, “The Method of Fundamental Solutions for the Inverse Heat Source Problem,” Engineering Analysis with Boundary Elements, Vol. 32, No. 3, 2008, pp. 216-222. http://dx.doi.org/10.1016/j.enganabound.2007.08.002

[14] L. Yan, F. L. Yang and C. L. Fu, “A Meshless Method for Solving an Inverse Spacewise-Dependent Heat Source Problem,” Journal of Computational Physics, Vol. 228, No. 1, 2009, pp. 123-136. http://dx.doi.org/10.1016/j.jcp.2008.09.001

[15] F. Yang, “The Truncation Method for Identifying an Unknown Source in the Poisson Equation,” Applied Mathematics and Computation, Vol. 22, 2011, pp. 9334-9339. http://dx.doi.org/10.1016/j.amc.2011.04.017

[16] F. Yang and C. L. Fu, “The Modified Regularization Method for Identifying the Unknown Source on Poisson Equation,” Applied Mathematical Modelling, Vol. 36, No. 2, 2012, pp. 756-763. http://dx.doi.org/10.1016/j.apm.2011.07.008

[17] Z. Yi and D. A. Murio, "Source Term Identification in 1-D IHCP," Computers \& Mathematics with Applications, Vol. 47, No. 12, 2004, pp. 1921-1933. http://dx.doi.org/10.1016/j.camwa.2002.11.025

[18] Z. Y. Zhao and L. You, “A Modified Tikhonov Regularization Method for Identifying an Unknown Source in the Heat Equation,” Acta Mathematica Scientia, 2012.

[19] A. Neubauer, "An a Posteriori Parameter Choice for Tikhonov Regularization in Hilbert Scales Leading to Optimal Convergence Rates,” SIAM Journal on Numerical Analysis, Vol. 25, No. 6, 1988, pp. 1313-1326. http://dx.doi.org/10.1137/0725074 
[20] H. W. Engl, M. Hanke and A. Neubauer, "Regularization of Inverse Problems,” Springer, Netherlands, 1996. http://dx.doi.org/10.1137/0725074

[21] F. Natterer, “Error Bounds for Tikhonov Regularization in Hilbert Scales,” Applicable Analysis, Vol. 18, No. 1-2, 1984, pp. 29-37. http://dx.doi.org/10.1080/00036818408839508 